\title{
Long-Ranged Attraction between Charged Polystyrene Spheres at Aqueous Interfaces
}

\author{
Wei Chen, ${ }^{1}$ Susheng Tan, ${ }^{2}$ Tai-Kai Ng, ${ }^{1}$ Warren T. Ford, ${ }^{2}$ and Penger Tong ${ }^{1, *}$ \\ ${ }^{1}$ Department of Physics, Hong Kong University of Science and Technology, Clear Water Bay, Kowloon, Hong Kong \\ ${ }^{2}$ Department of Chemistry, Oklahoma State University, Stillwater, Oklahoma 74078, USA
}

(Received 18 February 2005; published 15 November 2005)

\begin{abstract}
We report an optical and atomic force microscopic study of interactions between charged polystyrene spheres at a water-air interface. Optical observations of bonded particle clusters and formation of circular chainlike structures at the interface demonstrate that the interaction potential is of dipole origin. Atomic force microscope phase images show patchy domains on the colloidal surface, indicating that the surface charge distribution is not uniform as is commonly believed. Such surface heterogeneity introduces inplane dipoles, leading to an attraction at short interparticle distances.
\end{abstract}

DOI: 10.1103/PhysRevLett.95.218301

PACS numbers: 82.70.Dd, 61.20. $-\mathrm{p}$, 68.05.Gh

Coulomb repulsion between molecules of the same charge is a fundamental force necessary for the stability and structure formation of various macromolecules in aqueous solutions [1]. However, in some special circumstances, attractions are observed between like-charged species ranging from simple colloidal particles [2] to complex cytoskeletal filamentous actin [3] and DNA [4]. Because of its fundamental interest and important implications in colloid science and biology, the paradox of likecharge attractions has been under intensive theoretical scrutiny for many years [5]. Understanding of such attractions is needed for computer modeling, process control, and various engineering applications of colloids from soft materials to biotechnology. A careful examination of the experimental conditions reveals that the attractions occur when the charged particles (or macromolecules) and the screening counterions are in confined geometries such that the usual spherical symmetry of the counterion distribution is broken. For example, attractions between like-charged particles were found only when they are dispersed near a solid wall [2] or at a liquid interface [6,7].

Charged colloidal particles dispersed at an aqueous interface are stabilized by the Coulomb repulsion between the induced out-of-plane dipoles due to the asymmetric distribution of counterions in the aqueous phase $[8,9]$. The inset in Fig. 1 shows the surface charge on the particle and the asymmetric counterion clouds in water at an average separation of the Debye screening length $\lambda_{D}$ from the sphere's surface. If the charge distribution on the particle surface is uniform, a dipole moment pointing downward perpendicular to the interface forms with a magnitude [9] $P_{z} \simeq q_{0} \lambda_{D} / \sqrt{\epsilon}$, where $q_{0}$ is the effective charge carried by the particle and $\epsilon(\simeq 80)$ is the dielectric constant of water. A crucial assumption made for charged latex spheres is that the surface charge distribution of the particles is uniform. All such spheres have charge-stabilizing chemical functionality on their surfaces, but the actual distribution of the surface charge groups has not been examined systematically.
Recent experiments $[6,7]$ indicated that the interfacial particles also experience attractions, but the origin of such attractions remains illusive [7,10]. While these experiments revealed interesting mesostructure formation of particles at the interface, detailed information about the interaction potential $U(r)$ between the interfacial particles is rather limited. Experimental studies of $U(r)$ require well-controlled procedures to disperse the particles only onto the interface and to clean the interface and colloidal samples thoroughly. It is known that the interactions and dynamics of the interfacial particles are extremely sensitive to impurities at the interface.

In this Letter, we report a systematic experimental study of interactions between polystyrene (PS) latex spheres at a water-air interface. Two kinds of PS spheres are used: one has anionic (negative) carboxyl groups on the surface with diameter $d=1.1 \pm 0.02 \mu \mathrm{m}$ and (nominal) surface charge density $\sigma_{0}=12.5 \mu \mathrm{C} / \mathrm{cm}^{2}$, and the other has anionic sulfate groups on the surface with $d=1.0 \pm$ $0.03 \mu \mathrm{m}$ and $\sigma_{0}=2.8 \mu \mathrm{C} / \mathrm{cm}^{2}$. Both were purchased

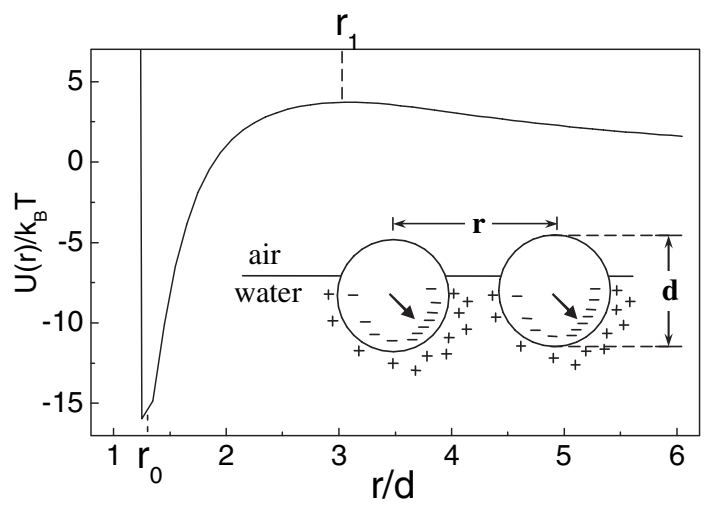

FIG. 1. Calculated interaction potential $U(r) / k_{B} T$ as a function of $r / d$. Inset shows schematic distributions of surface charge and counterions near the water-air interface. The lateral asymmetry of the counterion clouds gives rise to a horizontal dipole moment in the plane of the interface. 
from Interfacial Dynamics Corporation. The PS spheres are representative of charged particles commonly used in colloid science and have been used widely in experiments showing like-charge attractions at the interface [6] and near the solid wall [2].

The water-air interface is prepared using a homemade Teflon trough. The bottom of the trough has a hole sealed by a stainless steel cylinder with a thin glass cover slip on the bottom. The cylinder and the bottom glass slip serve as a sample cell with inner diameter $13 \mathrm{~mm}$ and height $1 \mathrm{~mm}$. The entire trough is placed on the sample stage of an inverted microscope (Leica DM-IRB), so that the motion of the interfacial particles can be viewed and recorded with a digital camera. The received aqueous latex samples are thoroughly cleaned with methanol via repeated centrifugation. Surface pressure measurements reveal that no detectable impurity is found in the cleaned latex samples. The particle-methanol solution is injected onto the interface by a syringe pump. The particles are found to be strongly bound to the interface, indicating that the vertical position of the particles is determined by a sharp surface energy minimum [8]. With the known surface tensions [8], we estimate that $2 / 3$ of the particle (by diameter) is immersed in water and $1 / 3$ is in air.

Two distinct particle configurations are observed at the interface. When the particle-methanol solution is injected onto the interface with low particle concentration and injection rate, a well dispersed layer of particles is obtained as shown in Fig. 2(a). The individual particles undergo vigorous Brownian motion and remain stable at the interface with various concentrations for days. Using a computer algorithm, we obtain the particle positions and calculate their pair correlation function $g(r)$, as shown in the inset in Fig. 3. For dilute particle concentrations, $g(r)$ is related to the interaction potential $U(r)$ through the Boltzmann equation $g(r)=\exp \left[-U(r) / k_{B} T\right]$, and the resulting $U(r) / k_{B} T$ is shown in Fig. 3. To avoid the crowding effect at finite concentrations, we calculate the many-body corrections to $U(r)$ using the hypernetted chain and PercusYevick approximations [11] and find that these corrections are negligible at the area fraction $n \simeq 0.12 \%$. Figure 3 shows that the measured $U(r)$ at large $r$ decays as $1 / r^{3}$ (solid curve).

When the particle-methanol solution is injected onto the interface with higher particle concentration and injection rate, the particles form stable bonded clusters, similar to those shown in Fig. 2(b), right after the evaporation of methanol. During the evaporation process, the particles at the interface may gain enough kinetic energy to overcome an energy barrier and form bonded clusters through an uncontrolled nonequilibrium process. This mechanism may also explain the mesostructure formation of interfacial particles reported from other experiments [6]. To test the mechanism, we start with an equilibrium configuration as shown in Fig. 2(a) and supply kinetic energy to the particles by periodically dilating the interface by pipetting a small amount of water $(\sim 0.5 \mathrm{ml})$ in and out of the aqueous
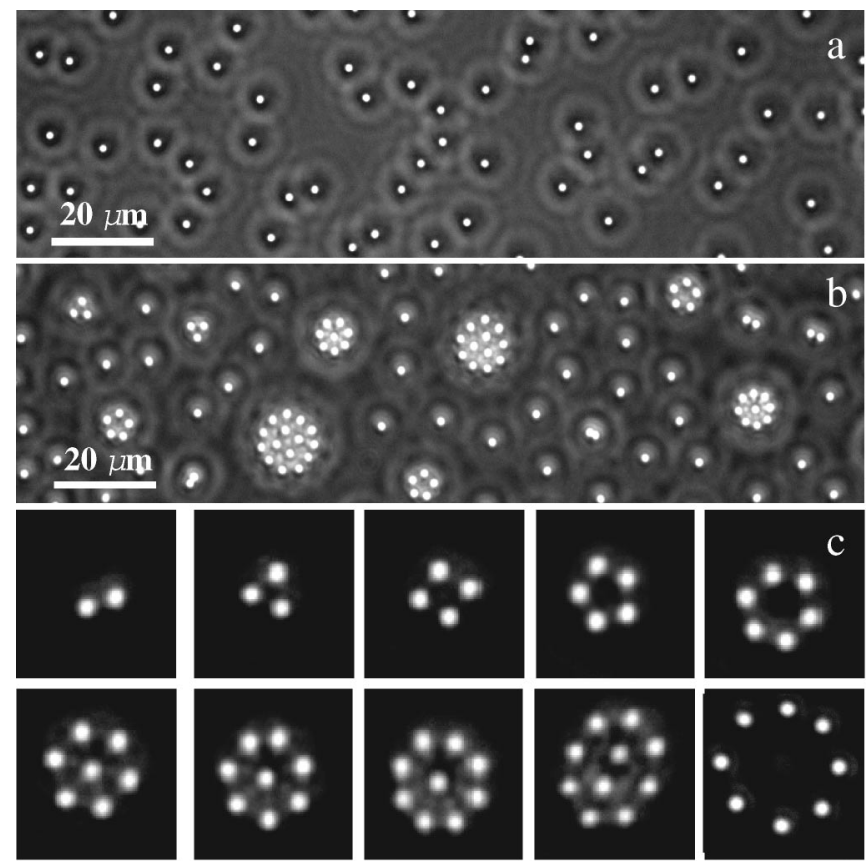

FIG. 2. (a) Equilibrium configuration of the interfacial particles at low surface coverage. Once dispersed onto the interface with a low surface coverage, these particles can be further concentrated by slowly pushing them into a smaller area with two Teflon barriers on the trough. (b) Formation of stable bonded particle clusters after the periodic dilation of the interface. (c) Enlarged images of the individual bonded particle clusters at the interface. All the clusters are composed of carboxyl-PS spheres except the last one, which is composed of sulfate-PS spheres. They carry fewer charges and show larger separation $r_{0}$ (particle size serves as a scale bar).

phase. After the periodic pipetting, the particle configuration changes from Fig. 2(a) to Fig. 2(b). The induced surface flow has a velocity $v \simeq 1 \mathrm{~cm} / \mathrm{s}$ and, thus, produces a kinetic energy of $m v^{2} / 2 \simeq 9 k_{B} T$ for the particles to overcome the energy barrier.

Figure 2(c) shows the configurations of different particle clusters formed after the periodic dilation of the interface. These particle clusters remain stable at the interface for days, indicating that the particles inside each cluster are trapped in a deep energy well. There is a significant separation between the neighboring particles within each cluster, making it different from particle aggregates in which all the particles are stuck together by van der Waals forces. The average separation between the particles is found to be $r_{0} \simeq 1.8 d$. Such a large particle separation suggests that there exists another strong repulsion between the particles at distances smaller than $r_{0}$. This repulsion balances the long ranged attraction, to be discussed below. From the above measurements, we arrive at the following qualitative picture for the interaction potential $U(r)$. As depicted in Fig. 1, it has an energy barrier with height $5-10 k_{B} T$ at $r_{1}$ and decays as $1 / r^{3}$ for larger $r\left(>r_{1}\right)$. For smaller particle separations, $U(r)$ has a deep attractive well of order $10 k_{B} T$ at $r_{0}$. For even smaller separations 


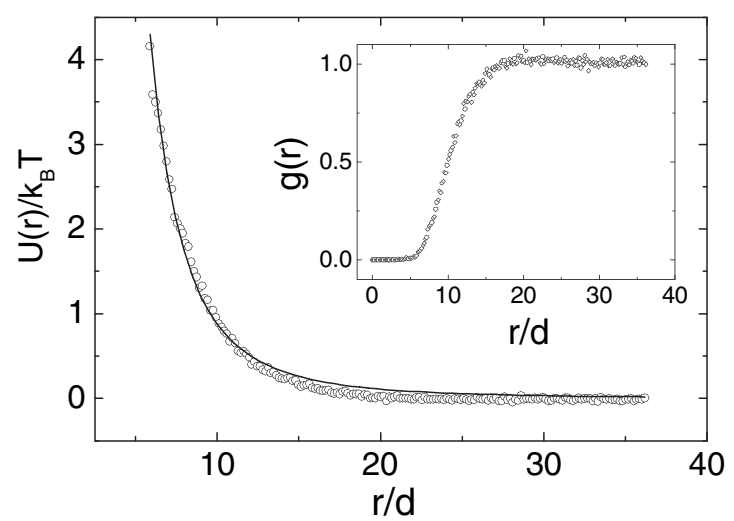

FIG. 3. Repulsive potential $U(r) / k_{B} T$ vs $r / d$ extracted from the measured $g(r)$ shown in the inset. Approximately $10000 \mathrm{im}-$ ages, each containing $\sim 60$ particles, taken at $\sim 1$ s intervals are used to calculate $g(r)$ with $\sim 100 \mathrm{~nm}$ spatial resolution. The area fraction occupied by the particles is $n \simeq 0.12 \%$. The solid curve shows the fitted function $U(r) / k_{B} T=879(d / r)^{3}$.

with $r \leqq d+\lambda_{D}$, the usual screened Coulomb repulsion is expected to dominate.

The potential $U(r)$ considers only the two-body interactions. The observed "Coulombic molecules" shown in Fig. 2(c) exhibit various molecular symmetries, which result from a unique many-body effect for the attraction between the interfacial particles. For example, we observe that the particles form many ringlike structures either after the periodic dilation of the interface or right after methanol evaporation. An example is given in the last panel in Fig. 2(c). Often, we find larger particle rings with a few extra particles moving freely at the center of the ring (these free particles do not belong to the particle cluster). The formation of such stable circular chains is a hallmark of dipolelike attractions under no external field. We believe that the various particle configurations shown in Fig. 2(c) result from minimizing the total electrostatic energy of the whole cluster. Some of the particle configurations shown in Fig. 2(c) were also observed in previous experiments [6,7].

As indicated in the inset in Fig. 1, one plausible cause for the formation of in-plane dipoles is that the charge distribution on the particle surface is not uniform. To examine the actual distribution of the surface charge groups, we conduct atomic force microscopy (AFM) measurements of surface topology of the individual PS spheres and phase shifts of the oscillating AFM tip. The AFM measurements are performed on a layer of PS spheres, which is obtained by depositing a drop of a dilute aqueous suspension of the particles on a newly cleaved mica substrate and drying at room temperature. The AFM topographic and phase images are obtained using an AFM (NanoScope IIIa) operating in tapping mode under ambient conditions $\left(24 \pm 1{ }^{\circ} \mathrm{C}\right.$, $50 \pm 5 \%$ relative humidity) and commercial silicon microcantilever probes with tip radius $5-10 \mathrm{~nm}$ and spring constant $2-5 \mathrm{~N} / \mathrm{m}$. Topographic and phase images are obtained simultaneously at a driving frequency of $120 \mathrm{kHz}$ for the probe oscillation, and the scanning rate of the images is set at $1 \mathrm{~Hz}$. To avoid damage to the particles, the AFM measurements are performed under light tapping conditions with a fixed set-point ratio $A_{\mathrm{sp}} / A_{0}=0.9$, where $A_{0}$ is the free oscillation amplitude (in air) and $A_{\mathrm{sp}}$ is a constant amplitude maintained during imaging.

Figure 4(a) shows the height image (two-dimensional) of a monolayer of the carboxyl-PS spheres. Figure 4(b) shows a magnified image (three-dimensional) of a sphere's top surface that is smooth down to a few nanometers. The phase images shown in Figs. 4(c) and 4(d) provide direct information about domains of different chemical composition of the particle surface. With the cantilever tip in the attractive regime $2-3 \mathrm{~nm}$ above a sphere's top surface, the phase delay of the oscillating cantilever is determined primarily by the van der Waals attraction between the cantilever tip and the almost flat top surface [12]. Because hydrophilic carboxyl-PS domains have a Hamaker constant different from that of hydrophobic neutral PS domains, the phase contrast images reveal the surface distribution of the ionizable carboxyl-PS groups [13]. Figure 4(c) shows the phase image simultaneously recorded with the height image shown in Fig. 4(b). We assign the yellow-green (lighter) regions with greater phase shift $(\sim 2 \pi / 7)$ to the carboxyl-PS domains. Patchy regions of carboxyl-PS domains of size $\sim 100 \mathrm{~nm}$ are found on the particle surface, indicating that the surface charge distribution of the PS spheres is not uniform as is commonly believed.

We have carried out extensive AFM measurements on five PS samples with different values of $d, \sigma_{0}$, and surface charge groups [14]. Figure 4(d) shows a phase image of the top surface of a sulfate-PS sphere, revealing similar patchy domains. The surface coverage of the patchy regions is reduced because the sulfate-PS spheres carry fewer sulfate-

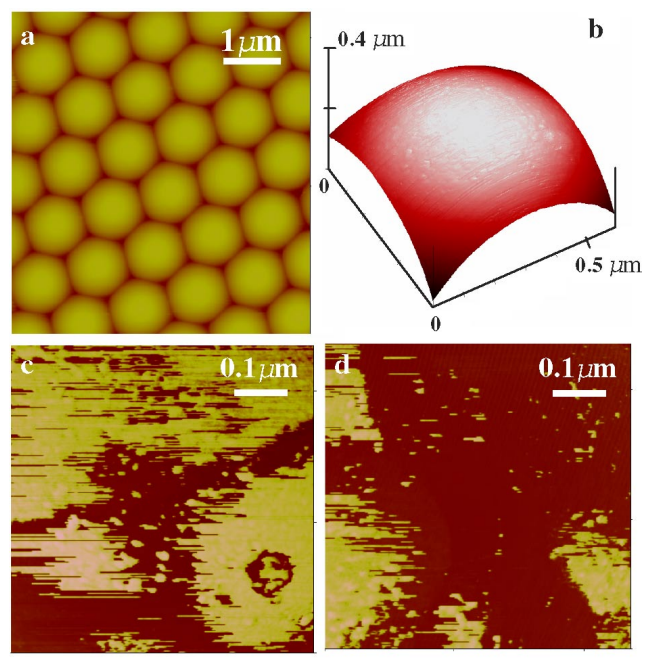

FIG. 4 (color online). (a),(b) AFM topographic images of the carboxyl-PS spheres with two different scan sizes. (c),(d) AFM phase images of the top surface of the carboxyl-PS and sulfatePS spheres, respectively. Typical phase contrast shown in (c),(d) is $\sim 2 \pi / 7$. 
PS groups (and, hence, fewer charges). The AFM measurements on the commercial samples and on laboratory synthesized PS samples of different surface chemistry [13] all reveal surface inhomogeneities similar to those shown in Fig. 4, suggesting that the observed surface heterogeneity is not simply a defect of a particular particle sample. Rather, it is a general behavior of the charged PS particles, which we attribute to surface phase separation between hydrophilic and hydrophobic chemical components.

The formation of chainlike structures shown in Fig. 2(c) together with the AFM measurements clearly demonstrate that the attraction between the interfacial particles is anisotropic in the plane of the interface. This finding prompts us to consider anisotropic attractions, such as electric dipole interactions, rather than the usual isotropic interactions, such as capillary forces and attractions resulting from wetting and surface roughness of the particles [7], for the interfacial particles. The size of the patchy domains of ionizable groups shown in Figs. 4(c) and 4(d) is too large to be averaged out spatially. Therefore, an in-plane dipole moment results with a magnitude $P_{\|} \simeq \alpha P_{z}$, where $0 \leq$ $\alpha \lesssim 1$ is a numerical factor that depends on the surface heterogeneity.

With both the in-plane and out-of-plane dipole contributions, the interaction potential between two (identical) interfacial particles can be written as (van der Waals forces not included) [1]

$$
U(r)=\frac{A e^{-r / \lambda_{D}}}{4 \pi \epsilon_{0} \bar{\epsilon} r}+\frac{P_{z}^{2}}{4 \pi \epsilon_{0} \bar{\epsilon} r^{3}}-\frac{P_{\|}^{2} f\left(\phi_{1}, \phi_{2}\right)}{4 \pi \epsilon_{0} \bar{\epsilon} r^{3}},
$$

where $\bar{\epsilon}$ is the effective dielectric contact at the interface, and $f\left(\phi_{1}, \phi_{2}\right)=\left\langle 3 \cos \phi_{1} \cos \phi_{2}-\cos \left(\phi_{2}-\phi_{1}\right)\right\rangle$ describes the alignment between two in-plane dipoles whose orientations are specified by the angles $\phi_{1}$ and $\phi_{2}$ with respect to the vector connecting the two particles. In Eq. (1), the first term represents the screened Coulomb repulsion (of amplitude $A$ ) between two charged spheres, the second term is the repulsion between two out-of-plane dipoles, and the third term is the attraction between two inplane dipoles. For a given temperature $T$, one can define a characteristic separation

$$
\lambda_{N}=\left[2 P_{\|}^{2} /\left(4 \pi \epsilon_{0} \bar{\epsilon} k_{B} T\right)\right]^{1 / 3},
$$

at which the attractive dipole interaction becomes equal to the thermal energy $k_{B} T$. For $r \geqslant \lambda_{N}, k_{B} T$ is large and we have $f\left(\phi_{1}, \phi_{2}\right) \simeq 0$, and, thus, $U(r)$ is dominated by the dipole-dipole repulsion. For $d+\lambda_{D} \lesssim r \lesssim \lambda_{N}$, the inplane dipoles are aligned in line by the attractive interaction, and, thus, $f\left(\phi_{1}, \phi_{2}\right) \simeq 2$. In this case, $U(r)$ becomes attractive if $2 P_{\|}^{2}>P_{z}^{2}$. At even smaller separations with $r \lesssim d+\lambda_{D}, U(r)$ is dominated by the usual screened Coulomb repulsion.

While Eq. (1) is correct only in the point-dipole limit, the characteristic $r$ dependence of $U(r)$ discussed above is quite general. To take the particle size effect into account, we calculate the electrostatic potential between two spheres by assuming that there is a layer of charge with surface charge density $\sigma(\theta, \phi)$ on the surface of a sphere immersed in water and another layer of charge $-\sigma(\theta, \phi)$ at distance $\lambda_{D}$ from the sphere's surface in water. For simplicity, we further assume that the sphere is half immersed in water, and $\sigma(\theta, \phi)$ takes a simple form $\sigma(\theta, \phi)=$ $\sigma_{0}\left[1+b \cos \left(\phi-\phi_{1}\right)\right]$, where $\sigma_{0}$ is the average surface charge density, $b$ is an adjustable parameter characterizing the surface heterogeneity, and $\theta$ and $\phi$ are, respectively, the angular variables in the spherical coordinates. In the calculation, we choose $\sigma_{0}=5.9 \mu \mathrm{C} / \mathrm{cm}^{2}, b=0.9$, and $\lambda_{D}=20 \mathrm{~nm}$. After a proper thermal averaging, we obtain $U(r) / k_{B} T$, and the final numerical result is shown in Fig. 1. While the surface charge distribution used is somewhat idealized, the resulting $U(r)$ proves instructive for our purpose. The characteristic features shown in Fig. 1 are all observed in the experiment with $r_{1} \sim \lambda_{N}$. Certainly, a more detailed modeling of $\sigma(\theta, \phi)$ is needed in order to calculate $U(r)$ for realistic PS spheres.

We are grateful for useful discussions and communications with D. Weitz, D. Grier, and J. Goodwin. This work was supported by the Research Grants Council of Hong Kong SAR (HKUST603504 and HKUST602803) and the National Science Foundation of the U.S. (EPS-0132534).

*Electronic address: penger@ust.hk

[1] J. Israelachvili, Intermolecular and Surface Forces (Academic, San Diego, 1991), 2nd ed.

[2] N. Ise et al., J. Chem. Phys. 78, 536 (1983); G. M. Kepler and S. Fraden, Phys. Rev. Lett. 73, 356 (1994); M.D. Carbajal-Tinoco et al., Phys. Rev. E 53, 3745 (1996); A. E. Laren and D. G. Grier, Nature (London) 385, 230 (1997); Y. Han and D. G. Grier, Phys. Rev. Lett. 91, 038302 (2003).

[3] T. A. Angelini et al., Proc. Natl. Acad. Sci. U.S.A. 100, 8634 (2003).

[4] W. M. Gelbart et al., Phys. Today 53, No. 9, 38 (2000).

[5] T. M. Squires and M. P. Brenner, Phys. Rev. Lett. 85, 4976 (2000); P. Attard, Curr. Opin. Colloid Interface Sci. 6, 366 (2001); D. G. Grier and Y. Han, J. Phys. Condens. Matter 16, S4145 (2004).

[6] F. Ghezzi and J. C. Earnshaw, J. Phys. Condens. Matter 9, L517 (1997); J. Ruiz-Garcia, R. Gamez-Corrales, and B. I. Ivlev, Phys. Rev. E 58, 660 (1998).

[7] M. G. Nikolaides et al., Nature (London) 420, 299 (2002).

[8] P. Pieranski, Phys. Rev. Lett. 45, 569 (1980).

[9] A. J. Hurd, J. Phys. A 18, L1055 (1985).

[10] M. Megens and J. Aizenberg, Nature (London) 424, 1014 (2003).

[11] S. H. Behrens and D. G. Grier, Phys. Rev. E 64, 050401(R) (2001).

[12] A. San Paulo and R. Garcia, Phys. Rev. B 64, 193411 (2001); R. W. Stark, G. Schitter, and A. Stemmer, Phys. Rev. B 68, 085401 (2003).

[13] S. Tan et al., Langmuir 21, 43 (2005).

[14] W. Chen et al. (unpublished). 